[0212-7199(2002) 19: 1; pp 7-10] ANALES DE MEDICINA INTERN Copyright $(9) 2002$ ARAN EDICIONES, S.L.

An. Med. Interna (Madrid) AN. MED. INTERNA (Madrid)
Vol. 19, N. ${ }^{1}$ 1, pp. 7-10, 2002

\title{
Anticuerpos frente al citoplasma de los neutrófilos (ANCA): correlaciones clínico-patológicas de una serie de 82 casos
}

\author{
J. M ${ }^{a}$ CALVO ROMERO, J. ROMERO REQUENA, J. C. ARÉVALO LORIDO, J. L. \\ RAMOS SALADO, E. DOBLARÉ CASTELLANO*, Mª L. VARGAS PÉREZ*, P. \\ BUREO DACAL, M. PÉREZ MIRANDA
}

\section{Servicio de Medicina Interna. *Sección de Inmunología. Hospital Universitario Infanta} Cristina. Badajoz

ANTINEUTROPHIL CYTOPLASMIC ANTIBODIES (ANCA): CLINI CAL-PATHOLOGICAL CORRELATES OF A SERIE OF 82 CASES

\section{RESUMEN}

Objetivo: Los anticuerpos frente al citoplasma de los neutrófilos (ANCA) se han descrito en una gran variedad de entidades además de en las vasculitis asociadas a ANCA [granulomatosis de Wegener (GW), poliangeítis microscópica (PAM) y síndrome de Churg-Strauss (SCS)] y en la glomerulonefritis rápidamente progresiva (GNRP) pauci-inmune. El objetivo de este trabajo es decribir los diagnósticos clínico-histológicos de una serie de pacientes con ANCA.

Material y métodos: Revisión retrospectiva de los pacientes con positividad para los ANCA mediante inmunofluorescencia indirecta en nuestro hospital entre enero de 1997 y diciembre de 1998.

Resultados: Identificamos 82 pacientes con ANCA Seis casos $(7,3 \%)$ presentaron un patrón C-ANCA, $19(23,2 \%)$ un patrón P-ANCA y $57(69,5 \%)$ un patrón atípico. Ocho pacientes $(9,7 \%)$ tuvieron una vasculitis asociada a ANCA (5 GW, 2 PAM y 1 SCS). Cuatro pacientes $(4,9 \%)$ tuvieron una GNRP pauci-inmune sin vasculitis sistémica. Un único caso $(1,2 \%)$ en nuestra serie presentó un patrón C-ANCA sin evidencia de vasculitis asociada a ANCA o GNRP pauci-inmune.

Conclusiones: El patrón atípico es el más frecuente en pacientes con ANCA. Una minoría de pacientes con ANCA tiene una vasculitis asocia da a ANCA (GW, PAM o SCS) o una GNRP pauci-inmune. La presencia de un patrón C-ANCA es excepcional en pacientes sin vasculitis asociada a ANCA o GNRP pauci-inmune.

PALABRAS CLAVE: Anticuerpos frente al citoplasma de los neutrófilos. ANCA. Correlaciones. Diagnóstico.

\section{ABSTRACT}

Objective: Antineutrophil cytoplasmic antibodies (ANCA) have been described in a great variety of diseases in addition to the ANCA-associa ted systemic vasculitis (Wegener's granulomatosis (WG), microscopic polyangiitis (MPA) and Churg-Strauss syndrome (CSS)) and the pauciimmune rapidly progressive glomerulonephritis (RPGN). The objective of this work is to describe the clinical-pathological diagnosis of a series of patients with ANCA.

Material and methods: Retrospective review of patients with positive ANCA by indirect immunofluorescence in our hospital between january 1997 and december 1998.

Results: We identify 82 patients with ANCA. Six cases (7.3\%) sho wed a C-ANCA pattern, 19 (23.2\%) a P-ANCA pattern and 57 (69.5\%) an atypical pattern. Eight patients (9.7\%) had an ANCA-associated sys temic vasculitis (5 WG, 2 MPA and 1 SCS). Four patients (4.9\%) had a pauci-immune RPGN without systemic vasculitis. One only case (1.2\%) in our series presented a C-ANCA pattern without evidence of an ANCAassociated systemic vasculitis or a pauci-immune RPGN.

Conclusions: The atypical pattern is the most frequent in patients with ANCA. A minority of patients with ANCA have an ANCA-associated systemic vasculitis (WG, MPA or SCS) or a pauci-immune RPGN. The occurrence of a C-ANCA pattern is exceptional in patients without an ANCA-associated systemic vasculitis or a pauci-immune RPGN.

KEY WORDS: Antineutrophil cytoplasmic antibodies. ANCA. Correla tes. Diagnosis.

Calvo Romero JMa, Romero Requena J, Arévalo Lorido JC, Ramos Salado JL, Doblaré Castellano E, Vargas Pérez. M ${ }^{a} L$, Bureo Dacal P, Pérez. Miranda M. Anticuerpos frente al citoplasma de los neutrófilos (ANCA): correlaciones clínico-patológicas de una serie de 82 casos. An Med Interna (Madrid) 2002; 19: 7-10.

\section{INTRODUCCIÓN}

Los anticuerpos frente al citoplasma de los neutrófilos (ANCA) son un grupo de autoanticuerpos dirigidos frente a constituyentes citoplasmáticos de los neutrófilos y de los monocitos (1-4). Los ANCA se asocian a la granulomatosis de Wegener (GW), la poliangeítis microscópica (PAM) y el síndrome de Churg-Strauss (SCS), que se agrupan bajo la denominación de vasculitis sistémicas de pequeño vaso asociadas a ANCA (1-5). También se ha descrito la positividad de los ANCA en la glomerulonefritis rápidamente progresiva (GNRP) pauci-inmune, en una gran variedad de enfermedades de naturaleza autoinmune, como el lupus eritematoso sistémico (LES), la artritis reumatoide, la esclerodermia, el síndrome de Sjögren, la polimiositis/dermatomiositis, la artritis crónica juvenil, la artritis reactiva, la enfermedad inflamatoria intesti-

Trabajo aceptado: 8 de octubre de 2001

Correspondencia: José María Calvo Romero. Héroes de Cascorro 9, $3^{\circ}$ A. 06004 Badjoz. 
nal crónica, la colangitis esclerosante primaria y la hepatitis autoinmune tipo I, y en ciertas infecciones (1-6). El objetivo del presente trabajo es determinar las correlaciones clínicopatológicas de nuestra reciente serie de pacientes con ANCA.

\section{MATERIAL Y MÉTODOS}

Realizamos una revisión retrospectiva de las historias clínicas de los pacientes de nuestro centro (Hospital Regional Universitario de la Comunidad Autónoma de Extremadura) con positividad para los ANCA en al menos una determinación entre enero de 1997 y diciembre de 1998. Se recogieron las características epidemiológicas y el diagnóstico clínicoanatomopatológico.

La determinación de los ANCA se realizó mediante inmunofluorescencia indirecta (IFI) sobre neutrófilos humanos fijados en etanol siguiendo el método descrito por Wiik (7), previa dilución del suero a 1:20. Las muestras positivas para ANCA fueron tituladas mediante IFI con diluciones seriadas dobles. Se consideró como título de la muestra la mayor dilución a la que se observara nítidamente el patrón de ANCA que se hubiera detectado en la primera determinación. Se consideró que existía positividad para los ANCA cuando el título de IFI fue igual o superior a 1:20.

Distinguimos 3 patrones de IFI: C-ANCA (citoplasmático), P-ANCA (perinuclear) y ANCA atípico. También se determinó la reactividad frente a la mieloperoxidasa (MPO) y la proteinasa 3 (PR3) mediante técnicas de ELISA (enzymelinked immunosorbent assay). En los pacientes con repetidas determinaciones de ANCA, se recogió el resultado en el momento de la evaluación diagnóstica inicial. El diagnóstico de las vasculitis sistémicas se realizó siguiendo los criterios utilizados por González-Gay y García-Porrúa (8). Los criterios utilizados para el diagnóstico de lupus eritematoso sistémico fueron los del American College of Rheumatology (9), y para el diagnóstico de la artritis reumatoide los de la American Rheumatism Association (10).

Las variables continuas se expresan como media \pm desviación standard. El análisis estadístico se realizó mediante el programa EPI-INFO 5,01 utilizando la prueba $\chi^{2}$ y el test exacto de Fisher, cuando alguno de los valores esperados fue menor de 5, para la comparación de proporciones y la prueba de la " $t$ " de Student para la comparación de medias. Se consideró estadísticamente significativo un valor de $\mathrm{p}<0,05$.

\section{RESULTADOS}

Identificamos 82 pacientes con positividad para los ANCA mediante IFI en el periodo estudiado. La edad media fue 59,8 $\pm 17,1$ años (rango 19-89 años). Cincuenta y cuatro pacientes $(65,8 \%)$ tuvieron una edad igual o superior a 60 años. El 54,9\% fueron mujeres. En la tabla I se detallan los diagnósticos de nuestra serie de pacientes con ANCA. El grupo de "otras glomerulonefritis" correspondió a: glomerulonefritis membranosa (3 casos), glomerulonefritis proliferativa mesangial ( 2 casos), glomerulonefritis membranoproliferativa ( 2 casos), y glomeruloesclerosis focal y segmentaria (2 casos). El grupo denominado "miscelánea" incluyó los siguientes diagnósticos: nefropatía hipertensiva (9 casos), nefropatía diabética ( 7 casos), nefropatía por AINES (3 casos), nefropatía
TABLA I

DIAGNÓ STICOSY PATRO NES DE NUESTRA SERIE

\begin{tabular}{lrrrr}
\hline & N=82 & C-Anca & P-Anca & Atípico \\
\hline Granulomatosis de W egener & $5(6,1 \%)$ & $40 \%$ & $60 \%$ & $0 \%$ \\
Poliangeítis microscópica & $2(2,4 \%)$ & $50 \%$ & $50 \%$ & $0 \%$ \\
Síndrome de Churg-Strauss & $1(1,2 \%)$ & $0 \%$ & $0 \%$ & $100 \%$ \\
GNRP pauci-inmune & $4(4,9 \%)$ & $50 \%$ & $50 \%$ & $0 \%$ \\
Otras glomerulonefritis & $9(11 \%)$ & $0 \%$ & $33,3 \%$ & $66,7 \%$ \\
LES & $8(9,8 \%)$ & $0 \%$ & $50 \%$ & $50 \%$ \\
Artritis reumatoide & $6(7,3 \%)$ & $0 \%$ & $0 \%$ & $100 \%$ \\
Síndrome de Sjögren primario & $3(3,6 \%)$ & $0 \%$ & $0 \%$ & $100 \%$ \\
Arteritis temporal/PMR & $3(3,6 \%)$ & $0 \%$ & $33,3 \%$ & $66,7 \%$ \\
Vasculitis leucocitoclástica & $2(2,4 \%)$ & $0 \%$ & $0 \%$ & $100 \%$ \\
Panarteritis nodosa & $1(1,2 \%)$ & $0 \%$ & $100 \%$ & $0 \%$ \\
Colitis ulcerosa & $4(4,9 \%)$ & $0 \%$ & $0 \%$ & $100 \%$ \\
Hepatitis crónica VHC & $4(4,9 \%)$ & $0 \%$ & $25 \%$ & $75 \%$ \\
Hepatitis crónica VHB & $2(2,4 \%)$ & $0 \%$ & $50 \%$ & $50 \%$ \\
M iscelánea & $28(34,1 \%)$ & $3,6 \%$ & $7,1 \%$ & $89,3 \%$ \\
\hline
\end{tabular}

GNRP: glomerulonefritis rápidamente progresiva.

LES: lupus eritematoso sistémico.

VHC: virus de la hepatitis $C$.

VHB: virus de la hepatitis $B$.

PM R: polimialgia reumática.

no especificada (2 casos), neoplasias (3 casos), fibrosis pulmonar idiopática ( 2 casos) y dermatitis neutrofílica (2 casos).

Seis casos del total $(7,3 \%)$ presentaron un patrón CANCA, $19(23,2 \%)$ un patrón P-ANCA y $57(69,5 \%)$ un patrón atípico. El título medio aproximado fue 1:80. Cuarenta pacientes $(48,8 \%)$ tuvieron un título igual o superior a 1:80, y sólo 14 pacientes $(17,1 \%)$ tuvieron un título igual o superior a $1: 160$. En la tabla I se describe la distribución de cada patrón en los distintos diagnósticos. En la tabla II se detallan los diagnósticos de los 40 pacientes con un título igual o superior a 1:80.

De los 6 casos con patrón C-ANCA, $4(66,7 \%)$ tuvieron reactividad frente a la PR3, 1 frente a la MPO y otro no presentó reactividad ni frente a la PR3 ni frente a la MPO. De los 19 casos con patrón P-ANCA, $10(52,6 \%)$ tuvieron reactividad frente a la MPO, 4 frente a la PR3 y 5 no presentaron reactividad frente a la MPO ni frente a la PR3. De los 57 casos con patrón atípico, sólo 1 caso $(1,7 \%)$ presentó reactividad frente a la PR3 y el resto no presentaron reactividad frente a la PR3 ni frente a la MPO. Un único caso $(1,2 \%)$ en nuestra serie presentó un patrón C-ANCA sin evidencia de vasculitis asociada a ANCA o GNRP pauci-inmune, y no presentó reactividad frente a la PR3 ni frente a la MPO.

Los 8 pacientes con LES presentaron anticuerpos antinucleares (ANA) a un título elevado (igual o superior a 1:640), 4 de ellos tuvieron un patrón P-ANCA (los 4 con reactividad frente a la MPO), y los otros 4 un patrón atípico (sin reactividad frente a la PR3 ni frente a la MPO). Los 3 casos de síndro- 


\section{TABLA II}

DIAGNÓSTICOS DE LOS PACIENTES CON TÍTULO DE ANCA $\geq 1: 80$

\begin{tabular}{lr}
\hline & \multicolumn{1}{c}{$\mathrm{N}=40$} \\
\hline M iscelánea & $11(27,5 \%)$ \\
Granulomatosis de W egener & $5(12,5 \%)$ \\
GNRP pauci-inmune & $4(10 \%)$ \\
Otras glomerulonefritis & $4(10 \%)$ \\
Artritis reumatoide & $4(10 \%)$ \\
Colitis ulcerosa & $4(10 \%)$ \\
Hepatis crónica VHC & $2(5 \%)$ \\
Arteritis temporal/PM R & $2(5 \%)$ \\
Síndrome de Churg-Strauss & $1(2,5 \%)$ \\
LES & $1(2,5 \%)$ \\
Hepatitis crónica VHB & $1(2,5 \%)$ \\
Vasculitis leucocitoclástica & $1(2,5 \%)$ \\
\hline
\end{tabular}

GNRP: glomerulonefritis rápidamente progresiva.

LES: lupus eritematoso sistémico.

VHC: virus de la hepatitis C.

VHB: virus de la hepatitis $B$

PMR: polimialgia reumática.

me de Sjögren presentaron ANA positivos a un título de 1:160, todos ellos con ANCA atípico y sin reactividad frente a la PR3 ni frente a la MPO. En ninguno del resto de nuestros pacientes se detectaron unos ANA positivos.

No encontramos correlación entre la edad y el título de ANCA $(r=-0,12)$. Tampoco encontramos diferencias significativas entre ambos sexos en el título de ANCA ni en la frecuencia de cada uno de los patrones de ANCA. Encontramos una cierta correlación positiva entre el título de ANCA y el título de ANA $(r=0,46)$ entre los pacientes con ANA positivos.

\section{DISCUSIÓN}

Los ANCA fueron inicialmente descritos en la década de los 80 en pacientes con GNRP o vasculitis sistémicas $(1,2)$. Con posterioridad, la presencia de ANCA se ha comunicado en una gran variedad de entidades además de en las denominadas vasculitis asociadas a ANCA (GW, PAM y SCS) y en la GNRP pauci-inmune sin vasculitis sistémica (1-6). Nuestro estudio ilustra la gran variedad de entidades que pueden asociarse a la presencia de ANCA. Sólo 8 pacientes $(9,7 \%)$ de nuestra serie de 82 pacientes con ANCA positivos (título de IFI igual o superior a 1:20) tuvieron una de las denominadas vasculitis asociadas a ANCA. Un título de IFI igual o superior a 1:20 es el habitualmente considerado para la positividad de los ANCA (7). Se ha sugerido que este límite se podría establecer en un título igual o superior a 1:40 con el objetivo de excluir falsos positivos (11). Sin embargo, en nuestra serie sólo 6 pacientes $(15 \%)$ de un total de 40 con un título de ANCA igual o superior a 1:80 tuvieron una vasculitis asocia- da a ANCA. Nuestros pacientes con un título de ANCA igual o superior a 1:80 también presentaron, al igual que en la serie completa, una gran variedad de diagnósticos (Tabla II). En nuestro estudio identificamos 4 casos de GNRP pauci-inmune sin vasculitis sistémica, entidad clásicamente asociada también a la presencia de ANCA $(1,2)$.

Se han descrito 3 patrones de ANCA en la IFI: citoplasmático, perinuclear y atípico $(3,4,12,13)$. Como en nuestra serie, el patrón C-ANCA o citoplasmático se debe habitualmente a anticuerpos dirigidos frente a la PR3, el patrón P-ANCA o perinuclear se debe más frecuentemente a anticuerpos dirigidos frente a la MPO, y el patrón atípico no suele presentar reactividad frente a la PR3 ni frente a la MPO $(3,4,12,13)$. Ocasionalmente el patrón C-ANCA puede corresponder a anticuerpos dirigidos frente a la MPO, y el patrón P-ANCA a anticuerpos dirigidos frente a la PR3, como también ocurrió en nuestra serie. La presencia de un patrón C-ANCA es excepcional en pacientes sin vasculitis asociada a ANCA o GNRP pauci-inmune $(3,4,12,13)$. Sólo 1 caso en nuestra serie presentó un patrón C-ANCA sin evidencia de vasculitis asociada a ANCA o GNRP pauci-inmune, además sin reactividad frente a la PR3 ni frente a la MPO. El patrón atípico corresponde habitualmente a una combinación de los patrones citoplasmático y perinuclear $(3,4,12,13)$. En nuestra serie el patrón atípico fue el más frecuente y se presentó en una gran variedad de diagnósticos, fundamentalmente en los incluidos en el grupo "miscelánea".

Un reciente consenso del International Group for Consensus Statement on Testing and Reporting of Antineutrophil Cytoplasmic Antibodies (11) ha establecido 4 patrones de ANCA: C-ANCA (patrón citoplasmático granular clásico con acentuación central o interlobular), C-ANCA atípico, PANCA y ANCA atípico. Este comité de expertos recomienda además la determinación habitual mediante técnicas de ELISA de la reactividad frente a la PR3 y la MPO. La limitación de la denominación C-ANCA a la descripción clásica de acentuación central e interlobular en la IFI mejora su correlación con la existencia de reactividad frente a la PR3 y con el diagnóstico de vasculitis asociada a ANCA (14).

La presencia de ANCA se ha descrito hasta en un $70 \%$ de los pacientes con LES y en un tanto por ciento menor de otras colagenosis como la esclerodermia, el síndrome de Sjögren y la conectivopatía indeferenciada (6). Corresponden casi sin excepción a un patrón P-ANCA o atípico, habitualmente sin reactividad frente a la PR3 ni frente a la MPO, y su significación clínica no ha sido elucidada (6). La positividad de los ANA puede dar lugar a resultados falsos positivos en la determinación de los ANCA, resultando en un patrón perinuclear (11). La fijación de los neutrófilos en formalina permite distinguir un verdadero patrón P-ANCA de los ANA (11), técnica que no se realizó en nuestros pacientes dado el carácter retrospectivo del estudio. No obstante, la frecuencia de reactividad frente a la MPO (50\%) en nuestros pacientes con LES y ANCA es muy superior a la descrita previamente del $1,4 \%$ (6), si bien nuestra serie de 8 casos es reducida.

En conclusión, sólo una minoría de pacientes con ANCA tiene una vasculitis asociada a ANCA o una GNRP pauciinmune, si bien la presencia de un patrón C-ANCA es excepcional en pacientes sin estas entidades. Además, no debe olvidarse que consideraciones técnicas y metodológicas de la determinación de los ANCA influyen en su sensibilidad y especificidad (11). 


\section{Bibliografía}

1. Davies DJ, Moran JE, Niall JF, Ryan GB. Segmental necrotising glomerulonephritis with antineutrophil antibody: possible arbovirus aetiology? BMJ 1982; 285: 606.

2. Falk RJ, Jennette JC. Antineutrophil cytoplasmic autoantibodies with specificity for myeloperoxidase in patients with systemic vasculitis and idiopathic necrotising and crescentic glomerulonephritis. N Engl J Med 1988; 318: 1651-7.

3. Nölle B, Specks U, Lüdemann J, Rohrbach MS, DeRemee RA, Gross WL. Anticytoplasmic autoantibodies: their immunodiagnostic value. Ann Intern Med 1989; 111: 28-40.

4. Hoffman GS, Specks U. Antineutrophil cytoplasmic antibodies. Arthritis Rheum 1998; 41: 1521-37.

5. Rao JK, Weinberger M, Oddone EZ, Allen NB, Landsman P, Feussner JR. The role of antineutrophil cytoplasmic antibody (c-ANCA) testing in the diagnosis of Wegener granulomatosis. A literature review and met-analysis. Ann Intern Med 1995; 123: 925-32.

6. Merkel PA, Polisson RP, Chang YC, Skates SJ, Niles JL. Prevalence of antineutrophil cytoplasmic antibodies in a large inception cohort of patients with connective tissue disease. Ann Intern Med 1997; 126: 866-73.

7. Wiik A. Delineation of a standard procedure for indirect immunofluorescence detection of ANCA. APMIS 1989; 97, 6: 12-3.
8. González-Gay MA, García-Porrúa C. Systemic vasculitis in adults in northwestern Spain, 1988-1997: Clinical and epidemiologic aspects. Medicine (Baltimore) 1999; 78: 292-308.

9. Tan EM, Cohen AS, Fries JF, et al. The 1982 revised criteria for the classification of systemic lupus erythematosus. Arthritis Rheum 1982; 25: 1271-7.

10. Arnett FC, Edworthy SM, Bloch DA, et al. The American Rheumatism Association 1987 revised criteria for the classification of rheumatoid arthritis. Arthritis Rheum 1988; 31: 315-24.

11. Savige J, Gillis D, Benson E, et al. International Consensus Statement on Testing and Reporting of Antineutrophil Cytoplasmic Antibodies (ANCA). Am J Clin Pathol 1999; 11: 507-13.

12. Lesavre P. Antineutrophil cytoplasmic autoantibodies antigen specificity. Am J Kidney Dis 1991; 18: 159-63.

13. Bang la Cour B, Wiik A, Møier-Madsen M, Baslund B. Clinical correlates and substrate specificities of antibodies exhibiting neutrophil nuclear activity a methodological study. J Immunol Methods 1995; 187: 287-95.

14. Wong RC, Silvestrini RA, Savige JA, Fulcher DA, Benson EM. Diagnostic value of classical and atypical antineutrophil cytoplasmic antibody (ANCA) immunofluorescence patterns. J Clin Pathol 1999; 52: 124-8. 